\title{
Correlation of Echocardiographic Parameters in Prone and Supine Positions in Normal Adults Using a Novel Approach.
}

\author{
Hesham Salah Taha ${ }^{1}$, Ahmed Mohamed ${ }^{1}$, Hossam Mahrous ${ }^{1}$, Osama Alsayed ${ }^{1}$, Mirna \\ Shaker $^{1}$, Hadeer Sayed ${ }^{1}$, Hussien Rizk ${ }^{1}$, and Mohamed Abdelwahab ${ }^{1}$ \\ ${ }^{1}$ Cairo University Kasr Alainy Faculty of Medicine
}

August 11, 2020

\begin{abstract}
Abstract Background: Transthoracic echocardiography (TTE) in prone position is challenging. Innovative use of transesophageal echocardiography (TEE) probe to perform TTE for such patients was described; but reproducibility and correlation of the TTE measurements by this technique with those obtained by the standard supine TTE study are still unknown. Methods: We enrolled 30 non-COVID-19 individuals, with a mean (SD) age 35 (10.9) years and 11 females, to study the agreement between the transthoracic measurements of the left ventricular (LV), left atrial (LA) and aortic dimensions obtained in prone position using an external TEE probe versus the standard supine position using the conventional TTE probe. Results: There were no significant differences between LV end-diastolic and end-systolic diameters, septal wall thickness, posterior wall thickness and aortic root dimensions in the prone versus the supine positions. While the mean ejection fraction $(\mathrm{EF})(60.3 \% \mathrm{vs} .63 .1 \%, \mathrm{P}=$ $0.014)$ and mean LA dimensions (1.8 vs. $1.9 \mathrm{~cm} / \mathrm{m} 2, \mathrm{P}<0.001)$ were significantly lower in the prone position. The mean time of scans was significantly longer in the prone as compared to the supine $(12.5$ vs 4.5 minutes, $\mathrm{P}<0.001)$. All supine studies had good quality while in the prone position 4 studies were of poor quality, and one was non-diagnostic. Conclusions: Assessment of cardiac dimensions and systolic function in the prone position using transthoracic TEE probe was feasible. LV and aortic dimensions agreed well with the standard TTE in supine position, however, LA dimensions and EF were lower in the prone position.
\end{abstract}

\section{Abstract}

Background: Transthoracic echocardiography (TTE) in prone position is challenging. Innovative use of transesophageal echocardiography (TEE) probe to perform TTE for such patients was described; but reproducibility and correlation of the TTE measurements by this technique with those obtained by the standard supine TTE study are still unknown.Methods: We enrolled 30 non-COVID-19 individuals, with a mean (SD) age 35 (10.9) years and 11 females, to study the agreement between the transthoracic measurements of the left ventricular (LV), left atrial (LA) and aortic dimensions obtained in prone position using an external TEE probe versus the standard supine position using the conventional TTE probe. Results: There were no significant differences between LV end-diastolic and end-systolic diameters, septal wall thickness, posterior wall thickness and aortic root dimensions in the prone versus the supine positions, while the mean ejection fraction $(\mathrm{EF})(60.3 \%$ vs. $63.1 \%, \mathrm{P}=0.014)$ and mean LA dimensions $(1.8$ vs. $1.9 \mathrm{~cm} / \mathrm{m} 2, \mathrm{P}<0.001)$ were significantly lower in the prone position. The mean time of scans was significantly longer in the prone as compared to the supine position (12.5 vs 4.5 minutes, $\mathrm{P}<0.001)$. All supine studies had good quality while in the prone position 4 studies were of poor quality, and one was non-diagnostic.Conclusions: Assessment of cardiac dimensions and systolic function in the prone position using transthoracic TEE probe was 
feasible. LV and aortic dimensions agreed well with the standard TTE in supine position, however, LA dimensions and EF were lower in the prone position.Keywords: COVID-19, transthoracic echocardiography, transesophageal, prone position, cardiac dimensions, systolic function.

\section{Introduction}

Preexisting cardiovascular diseases are linked to more severe COVID-19 infection. Additionally, cardiovascular complications can be a significant contributor to the mortality associated with this disease.1, 2 It has been shown that prone ventilation can improve oxygenation in patients with acute respiratory distress syndrome (ARDS) secondary to COVID-19.3 Echocardiography used to assess their cardio-circulatory status, may be very challenging in patients kept in the prone position using conventional transthoracic echocardiography (TTE). Transesophageal echocardiography (TEE) is a traditional solution that can be performed in the prone position and was reported to be a safe procedure in ARDS patients.4 However, TEE carries a heightened risk of spread of the COVID-19, as it can promote the aerosolization of a large amount of virus.5 Additionally, acute access to TEE study is a logistic problem particularly that it requires highly trained professionals on image acquisition and interpretation. Ugalde et al.6 and Giustiniano et al.7 had previously described techniques to obtain TTE using the conventional probe during prone position ventilation, but those techniques were demanding and required special positioning and preparations. Lately, Marvaki A et al.8 described an innovative use of the TEE probe to perform a transthoracic study on patients with COVID-19, who were invasively ventilated in the prone position. This was feasible and of acceptable diagnostic value. However, the reproducibility and correlation of the echocardiographic measurements obtained by this novel technique with those obtained by the standard transthoracic study are still unknown. The aim of this work was to compare the basic left ventricular (LV), left atrial (LA) and aortic (Ao) dimensions obtained by TTE imaging in the prone position using a TEE probe versus the standard supine position using the conventional TTE probe in apparently healthy adults.

\section{Patients and methods}

Thirty apparently healthy non-COVID-19 patients, referred for routine TTE at the outpatient clinic of cardiology department at Cairo University hospital, were prospectively enrolled in July 2020. Each participant was examined in the same sitting, both in the prone position using a TEE probe and in the standard supine position using the conventional TTE probe, by two independent operators. All scans were performed with a Philips Affiniti 50C machine using the S4-2 TTE probe and the X7-2t TEE probe (Philips Healthcare, Andover, MA). The basic LV internal dimensions, wall thickness, and ejection fraction (EF), in addition to left atrial (LA) and aortic root (AoR) dimensions were obtained carefully in either position from electrocardiographically (ECG) - gated two-dimensional (2D) images of parasternal long axis view with great attention to be perpendicular to LV/LA long axis.

The standard supine position studies were done according to the American Society of Echocardiography (ASE) and European Association of Cardiovascular Imaging (EACVI) recommendations.9 Regarding the prone study the same innovative technique recently described by Marvaki A et al. ${ }^{8}$ was adopted. In this technique, the operator was standing on the left side of the patient at the level of the head, facing the patient's right side. The probe was anchored to patient's chest wall against bed mattress. The tip of the probe lubricated by gel was placed in the standard left parasternal position in the third or fourth left intercostal space. The transducer was facing upwards and was supported underneath by operator's left hand. The probe shaft was held with the right hand to allow rotation. Images of the long axis of the heart were obtained at zero angle as in the conventional TTE parasternal long-axis view (Figure 1), while images of the short axis view were obtained by increasing the angle to $60-120$ degrees, usually at the level of the mitral 
valve. By rotating the probe clockwise, the mid-papillary level was obtained (Figure 2), while great vessels level was obtained by anticlockwise rotation.

The scan time was measured, and the image quality was graded as poor, fair, good or non-diagnostic. The quality of the images acquired by the TEE probe in the prone position was compared to that of the images obtained by TTE probe in the supine position.

Figure 1. Transthoracic parasternal long axis view obtained in the prone position by the TEE probe at angle $0^{\circ}$

A: Parasternal long axis viewB: 2D measurements of LV internal dimensions and wall thickness at end diastole C: 2D measurements of LV internal dimensions at end systole

Figure 2. Transthoracic parasternal short axis view obtained in the prone position by the TEE probe at angle $77^{\circ}$

A: At the level of mitral valve leafletsB: At the level of papillary muscles obtained by clockwise rotationC: At the level of great vessels obtained by counterclockwise rotation

\section{Statistical analysis}

Continuous variables were presented as means \pm standard deviation (SD) or medians with interquartile range (IQR) and compared with Student's t-tests or the Mann-Whitney U test. Categorical variables were reported as numbers and percentages and compared with the Chi- square or Fisher exact test (for cells $<$ $5)$.

A Pearson correlation coefficient was computed to assess the relationship between each of the LV dimensions, $\mathrm{EF}$, aortic root and LA dimensions indexed to body surface area in both the prone and the supine positions. An independent-samples t-test was conducted to compare differences in the mean diameter for each parameter between both positions. Bland-Altman plots were performed to show accuracy and agreement between each of LV- end diastolic diameter (LVEDD), LV- end systolic diameter (LVESD), septal wall thickness (SWT), posterior wall thickness (PWT), and aortic root dimensions (AoR) in the prone position vs the standard supine position.

Two-tailed $\mathrm{p}$ values $<0.05$ were considered significant. Statistical analyses were performed by using the IBM Statistical Package for Social Sciences (SPSS) for Windows version 25.0 (IBM SPSS Inc. USA).

\section{Results}

Overall, 30 non-COVID-19 patients were enrolled in our study. Mean (SD) age of patients was 35 (10.9) years and 11 patients $(36.7 \%)$ were females. One patient had hypertension, 1 patient had non-insulin dependent diabetes mellitus (DM) and 8 patients were smokers. Mean (SD) BMI was $26.4(5.9) \mathrm{kg} / \mathrm{m} 2$ and mean (SD) BSA was $1.8(0.2) \mathrm{m} 2$.

There were no significant differences between LVEDD, LVESD, SWT, PWT and AoR dimensions in the prone vs the supine positions. While there was a significant difference between the mean ejection fraction (60.3\% vs $63.1 \%, \mathrm{P}=0.014)$ and mean LA dimensions $(1.8[0.2]$ vs $1.9(0.2) \mathrm{cm} / \mathrm{m} 2, \mathrm{P}<0.001)$ in the prone position as compared to the supine position (table 1). 
Table 1. Comparing differences between each of the LV dimensions, ejection fraction, aortic root and LA dimensions in the prone vs supine positions.

\begin{tabular}{lllll}
\hline & $\begin{array}{l}\text { Supine } \\
\text { Mean (SD) }\end{array}$ & Prone Mean (SD) & $\begin{array}{l}\text { Mean differences } \\
\text { (SD) }\end{array}$ & P value \\
\hline LVEDS cm/m $\mathbf{2}$ & $2.7(0.4)$ & $2.6(0.4)$ & $-0.13(0.26)$ & 0.051 \\
LVESD cm/ m2 & $1.8(0.3)$ & $1.7(0.3)$ & $-.041(0.20)$ & 0.284 \\
SWT cm/m2 & $0.5(0.1)$ & $0.4(0.1)$ & $-0.01(0.080)$ & 0.575 \\
PWT cm/m2 & $0.4(0.1)$ & $0.4(0.1)$ & $-0.01(0.104)$ & 0.562 \\
EF $\mathbf{~ \% ) ~}$ & $63.1(5.0)$ & $60.3(4.3)$ & $-2.40(0.95)$ & 0.014 \\
Aortic root & $1.6(0.2)$ & $1.5(0.2)$ & $-0.09(0.26)$ & 0.073 \\
$\mathbf{c m} / \mathbf{m} \mathbf{2}$ & & & & \\
$\mathbf{L A ~} \mathbf{~ c m} / \mathbf{m} \mathbf{2}$ & $1.9(0.2)$ & $1.8(0.2)$ & $-0.15(0.198)$ & $<0.001$ \\
\hline
\end{tabular}

LVEDD: Left ventricular end diastolic diameter. LVESD: Left ventricular end systolic diameter. dSWT: Septal wall thickness in diastole. dPWT: posterior wall thickness in diastole. EF: Ejection fraction. AoR: Aortic root. LA: Left atrium.

There was a significant positive correlation between each of the measurements in the prone and the supine positions, as shown in Table 2. LVEDD and LVESD showed the strongest correlation between their measurements in the prone and the supine positions $(\mathrm{r}=0.732$ and 0.728 respectively, $\mathrm{P}<0.001$ for both $)$, as shown infigures 3 and 4, while EF and AoR dimensions showed the lowest correlation $(\mathrm{r}=0.386$ and 0.382 respectively).

Table 2. Correlation between LV dimensions, ejection fraction, aortic root and LA dimensions in the prone and supine positions.

\begin{tabular}{lllll}
\hline & $\begin{array}{l}\text { Supine } \\
\text { Mean (SD) }\end{array}$ & $\begin{array}{l}\text { Prone } \\
\text { Mean (SD) }\end{array}$ & Pearson correlation coefficient $(\mathbf{r})$ & P value \\
\hline LVEDD cm/m2 & $2.7(0.4)$ & $2.6(0.4)$ & 0.732 & $<0.001$ \\
LVESD cm/m2 & $1.8(0.3)$ & $1.7(0.3)$ & 0.728 & $<0.001$ \\
dSWT cm/m2 & $0.5(0.1)$ & $0.4(0.1)$ & 0.458 & 0.013 \\
dPWT cm/m2 & $0.4(0.1)$ & $0.4(0.1)$ & 0.497 & 0.006 \\
EF $\mathbf{\%})$ & $63.1(5.0)$ & $60.3(4.3)$ & 0.386 & 0.039 \\
AoR $\mathbf{~ c m} / \mathbf{m} \mathbf{2}$ & $1.6(0.2)$ & $1.5(0.2)$ & 0.382 & 0.041 \\
LA $\mathbf{~ m ~} / \mathbf{m} \mathbf{2}$ & $1.9(0.2)$ & $1.8(0.2)$ & 0.556 & 0.002 \\
\hline
\end{tabular}


LVEDD: Left ventricular end diastolic diameter. LVESD: Left ventricular end systolic diameter dSWT: Septal wall thickness in diastole. dPWT: posterior wall thickness in diastole. EF: Ejection fraction. AoR: Aortic root. LA: Left atrium.

Figure 3. Correlation between LVEDD in the prone position (Y axis) and the corresponding measurement in the supine position $(\mathrm{x}$ axis) $(\mathrm{r}=0.732 . \mathrm{P}<0.001)$

Figure 4. Correlation between LVESD in the prone position ( $\mathrm{Y}$ axis) and the corresponding measurement in the supine position $(\mathrm{x}$ axis $)(\mathrm{r}=0.728 . \mathrm{P}<0.001)$

Bland-Altman plots were performed to show accuracy and agreement between each of LVEDD, LVESD, SWT, PWT, and AoR dimensions in the prone position versus the standard supine position. The differences between each of the measurements were plotted against the averages of the measurements in both positions. The mean of the difference and the upper and lower limits of differences were estimated.

All measurements for LVEDD, LVESD, SWT, PWT and AoR dimensions in the prone position were accurate and agreed well with their corresponding measurements in the supine

position with very small mean differences, as shown in Table 3. Measurements of SWT and PWT dimensions, followed by LVES dimensions, in the prone position were the most accurate, and agreed well with their corresponding measurements in the supine position, as they showed the smallest mean differences as shown in Figures 5, 6 and 7respectively. LVED dimension in the prone position was the farthest from its corresponding measurement in the supine position (as it showed the largest mean difference).

Table 3. Differences between each of the LVEDD, LVESD, and aortic root dimensions in the prone versus supine positions derived from Bland-Altman plots

\begin{tabular}{llll}
\hline & $\begin{array}{l}\text { Mean differences } \\
\text { (SD) between the } \\
\text { prone and the } \\
\text { supine positions }\end{array}$ & $\begin{array}{l}\text { Lower limit of } \\
\text { difference }\end{array}$ & $\begin{array}{l}\text { Upper limit of } \\
\text { difference }\end{array}$ \\
\hline LVEDD cm/m2 & $-0.13(0.26)$ & -0.64 & 0.39 \\
LVESD cm/ $\mathbf{m} 2$ & $-0.04(0.20)$ & -0.44 & 0.35 \\
dSWT $\mathbf{~ c m} / \mathbf{m} \mathbf{2}$ & $-0.01(0.08)$ & -0.16 & 0.15 \\
dPWT $\mathbf{~ c m} / \mathbf{m} \mathbf{2}$ & $-0.01(0.10)$ & -0.22 & 0.19 \\
AoR $\mathbf{~ m ~} / \mathbf{m} \mathbf{2}$ & $-0.09(0.26)$ & -0.59 & 0.42 \\
\hline
\end{tabular}

LVEDD: Left ventricular end diastolic diameter. LVESD: Left ventricular end systolic diameter. dSWT: Septal wall thickness in diastole. dPWT: posterior wall thickness in diastole. AoR: Aortic root.

Figure 5. Bland-Altman plot for difference between SWT in the prone versus the supine position

Figure 6. Bland-Altman plot for difference between PWT in the prone versus the supine positions

Figure 7. Bland-Altman plot for difference between LVESD in the prone versus the supine positions 
The mean time of scan was significantly longer in the prone position as compared to the supine position (12.5 vs 4.5 minutes, $\mathrm{p}<0.001$ ). In the supine position, the standard echocardiographic views (PLAX, PSAX at the level of great vessels, mitral valve and papillary muscles, apical and subcostal views) were feasible in all patients. While in the prone position the PLAX view was feasible in 29 patients, the PSAX view at the level of the the great vessels, mitral valve and papillary muscles were feasible in 15, 27 and 20 patients respectively.

There was statistically significant difference in the quality of scans in both positions, with the supine position having good quality scans as compared to the prone position $(\mathrm{p}<0.001)$. All supine studies had good quality, while in the prone position 2, 23 and 4 studies had good, fair and poor qualities respectively. Only in one study it was non-diagnostic in the prone position due to obesity (BMI was $35.5 \mathrm{~kg} / \mathrm{m} 2$ ). Figure 8 is a graphic demonstration of the frequencies of the scan quality.

\section{Figure 8. Frequencies of scans quality in the prone position studies}

\section{Discussion}

Coronavirus disease 19 (COVID- 19) pandemic has affected the medical practice in general and the cardiology practice in particular.

It has been shown that prone ventilation can improve oxygenation in patients with acute respiratory distress (ARDS) secondary to COVID-19.1 The use of TTE to assess the cardiovascular complications of COVID-19 is very challenging on patients kept in the prone position.2

A recently published study by Marvaki et al.8 has showed the feasibility of an innovative use of a TEE probe to perform TTE on patients with COVID-19 who were invasively ventilated in the prone position. They have concluded that the innovative use of a TEE probe to perform TTE in the prone positioned and invasively ventilated ICU patients, was feasible and of diagnostic quality in most cases, and could be an alternative to conventional TTE on prone patients.

The findings of our study confirmed that the unconventional use of TEE probe to perform TTE with patients kept in the prone position was feasible and reproducible. There was generally good agreement between the linear 2D measurements of the left ventricle by this innovative technique and the corresponding measurements obtained by the standard supine TTE. In this study we recruited apparently healthy adult individuals who were examined by both techniques in the same sitting by the same echocardiography machine thus it is considered as a proof of concept that was previously proposed by Marvaki et al. ${ }^{8}$

Small, yet statistically significant differences, were noticed in the LV EF and LA anteroposterior diameter between the two techniques, being lower in the prone position. This could be explained by the effect of changing the direction of the transverse gravitational stress on cardiovascular variables. Also, foreshortened views in the prone position may explain the smaller left atrial dimensions in this position.

Bettina Pump et al.10 studied the physiological effects of the supine, prone, and lateral positions on cardiovascular and renal variables in humans. They concluded that the prone position reduced the stroke volume by $16 \%$, and increased the sympathetic nervous system activity as evidenced by: increased heart rate, total peripheral vascular resistance and plasma concentration of norepinephrine. These effects may have been caused by some compression of the thorax leading to impediment of the arterial filling and thus inhibition of the arterial baroreflexes. But no significant difference in the left atrial diameter was noted on comparing the effects of the supine with the prone positions.

Also on performing agreement analysis, the LVEDD in the prone position was the farthest from its corresponding measurement in the supine position (as it showed the largest mean difference). This was concordant with the observations noted by Wolfgang M. Schaefer et al,11 who investigated the effect on LV volumes, 
$\mathrm{EF}$, and heart rate in the prone versus the supine positions during gated 99mTc-Sestamibi single photon emission computer tomography (SPECT). They found that the end-diastolic volume and stroke volume were significantly lower in the prone acquisitions than in the corresponding supine measurements; however, the end-systolic volume and LV EF did not differ significantly.

In the prone position, we were able to acquire parasternal views (either long axis or short axis) in most of our patients (29/30 patients) compared to the original study, that reported diagnostic image quality in 17 out of 21 patients. In the majority of patients, the image quality was fair in the prone position, with significantly longer scan time than in the supine position. Most studies required an assistant to handle the echocardiography machine while the main operator was controlling the probe.

However, this novel technique allowed, in less than a quarter of an hour, an informative assessment of global LV systolic function, right ventricular out flow tract, pulmonary artery, and also rapid screening of the valvular morphology and function with exclusion of any valve-related masses, intracardiac thrombi or pericardial effusion. This technique was not only reproducible, but also offered a relatively comfortable and safe transthoracic examination for the patients in this challenging prone position.

\section{Conclusion}

The novel use of the TEE probe for transthoracic examination of patients in the prone position is feasible and reproducible. There was a good agreement between the 2D linear measurements of the LV and AoR in the prone position compared to the corresponding measurements obtained in the supine position by the standard transthoracic technique. However, the EF was lower, and left atrial anteroposterior diameter was smaller in the prone position.

\section{Acknowledgement of grant support}

This research received no specific grant from any funding agency in the public, commercial, or not-for-profit sectors.

\section{Conflict of interest}

The authors declare that there is no conflict of interest.

\section{Ethical approval}

All procedures performed in studies involving human participants were in accordance with the ethical standards of the institution and with the 1964 Helsinki declaration and its later amendments.

\section{Informed consent}

The informed consent was obtained from all participants included in the study.

\section{References}

1. Driggin E, Madhavan MV, Bikdeli B, Chuich T, Laracy J, Biondi-Zoccai G, Brown TS, Der Nigoghossian C, Zidar DA, Haythe J, Brodie D. Cardiovascular considerations for patients, health care workers, 
and health systems during the COVID-19 pandemic. Journal of the American College of Cardiology. 2020 May 12;75(18):2352-71.

2. Ruan Q, Yang K, Wang W, Jiang L, Song J. Clinical predictors of mortality due to COVID-19 based on an analysis of data of 150 patients from Wuhan, China. Intensive care medicine. 2020 May;46(5):846-8.

3. Gibson PG, Qin L, Puah S. COVID-19 ARDS: clinical features and differences to "usual" pre-COVID ARDS. Med J Aust. 2020 Apr 24.

4. Dessap AM, Proost O, Boissier F, Louis B, Campo FR, Brochard L. Transesophageal echocardiography in prone position during severe acute respiratory distress syndrome. Intensive care medicine. 2011 Mar $1 ; 37(3): 430-4$.

5. Kirkpatrick JN, Mitchell C, Taub C, Kort S, Hung J, Swaminathan M. ASE statement on protection of patients and echocardiography service providers during the 2019 novel coronavirus outbreak. Journal of the American College of Cardiology. 2020 Apr 6.

6. Ugalde D, Medel JN, Romero C, Cornejo R. Transthoracic cardiac ultrasound in prone position: a technique variation description. Intensive care medicine. 2018 Jun;44(6):986-

7. Giustiniano E, Padua E, Negri K, Bragato RM, Cecconi M. Echocardiography during prone-position mechanical ventilation in patients with COVID-19: a proposal for a new approach. Journal of the American Society of Echocardiography. 2020 Jul 1.

8. Marvaki A, Papachristidis A, Nakou E, Toth E, O'Gallagher K, Fisher R, Shah AM, Monaghan MJ. Innovative transthoracic echocardiographic imaging on prone ventilated patients with COVID-19 using a transesophageal probe. Jacc. Cardiovascular Imaging. 2020 Jun 23.

9. Lang RM, Badano LP, Mor-Avi V, Afilalo J, Armstrong A, Ernande L, Flachskampf FA, Foster E, Goldstein SA, Kuznetsova T, Lancellotti P. Recommendations for cardiac chamber quantification by echocardiography in adults: an update from the American Society of Echocardiography and the European Association of Cardiovascular Imaging. European Heart Journal-Cardiovascular Imaging. 2015 Mar 1;16(3):233-71.

10. Pump B, Talleruphuus U, Christensen NJ, Warberg J, Norsk P. Effects of supine, prone, and lateral positions on cardiovascular and renal variables in humans. American Journal of Physiology-Regulatory, Integrative and Comparative Physiology. 2002 Jul 1;283(1):R174-80.

11. Schaefer WM, Lipke CS, Kühl HP, Koch KC, Kaiser HJ, Reinartz P, Nowak B, Buell U. Prone versus supine patient positioning during gated $99 \mathrm{mTc}$-sestamibi SPECT: effect on left ventricular volumes, ejection fraction, and heart rate. Journal of Nuclear Medicine. 2004 Dec 1;45(12):2016-20. 

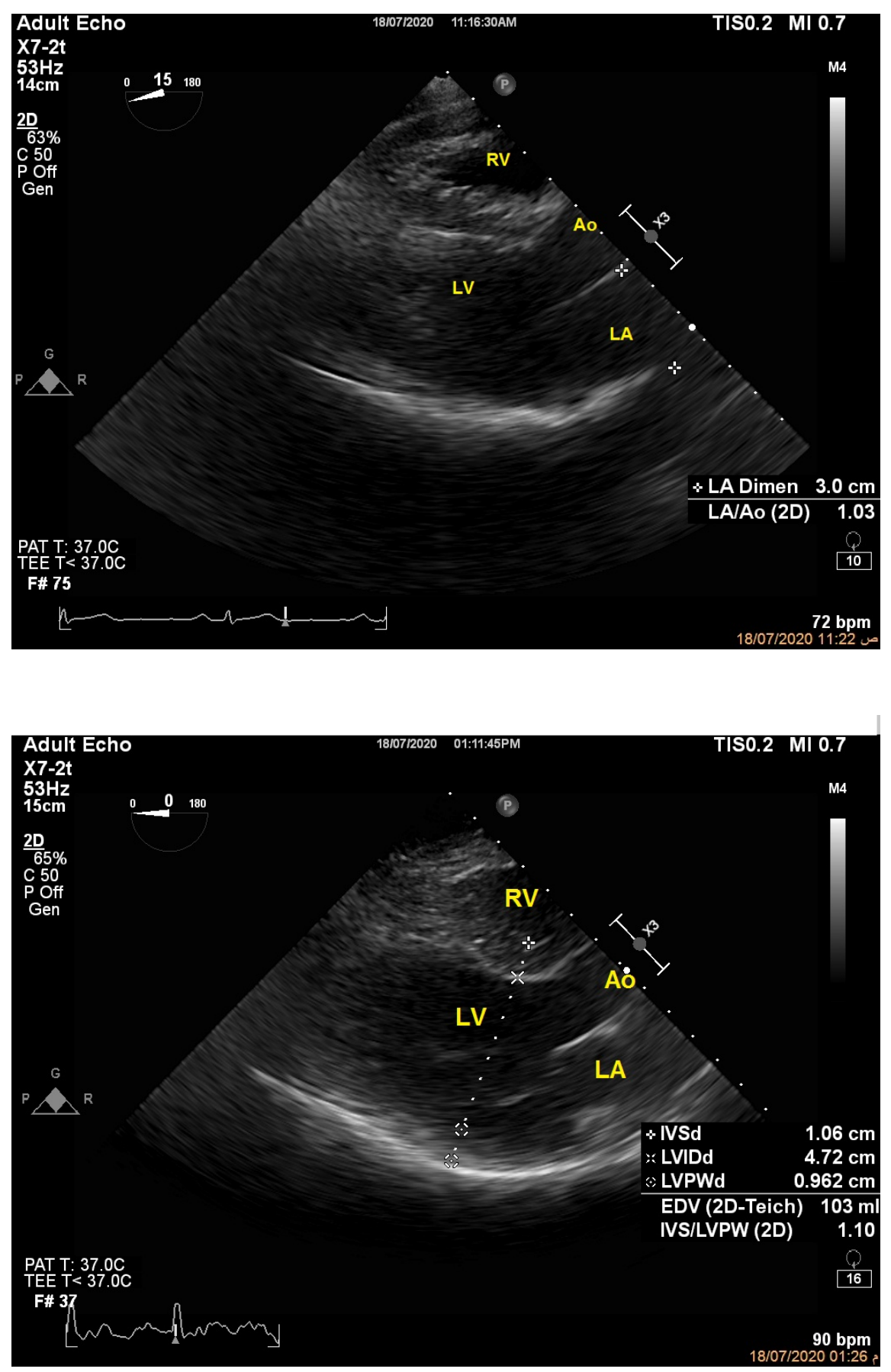

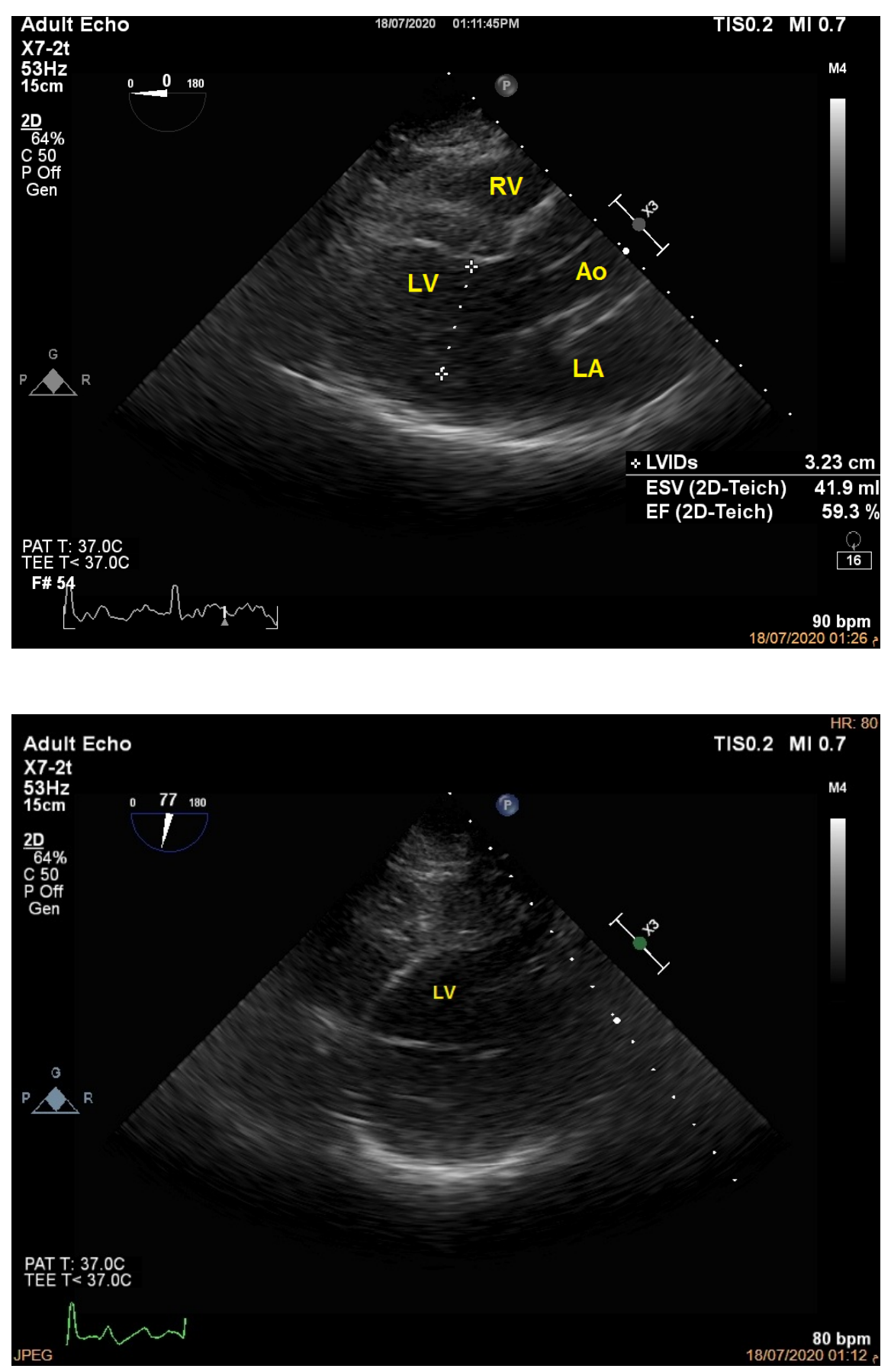

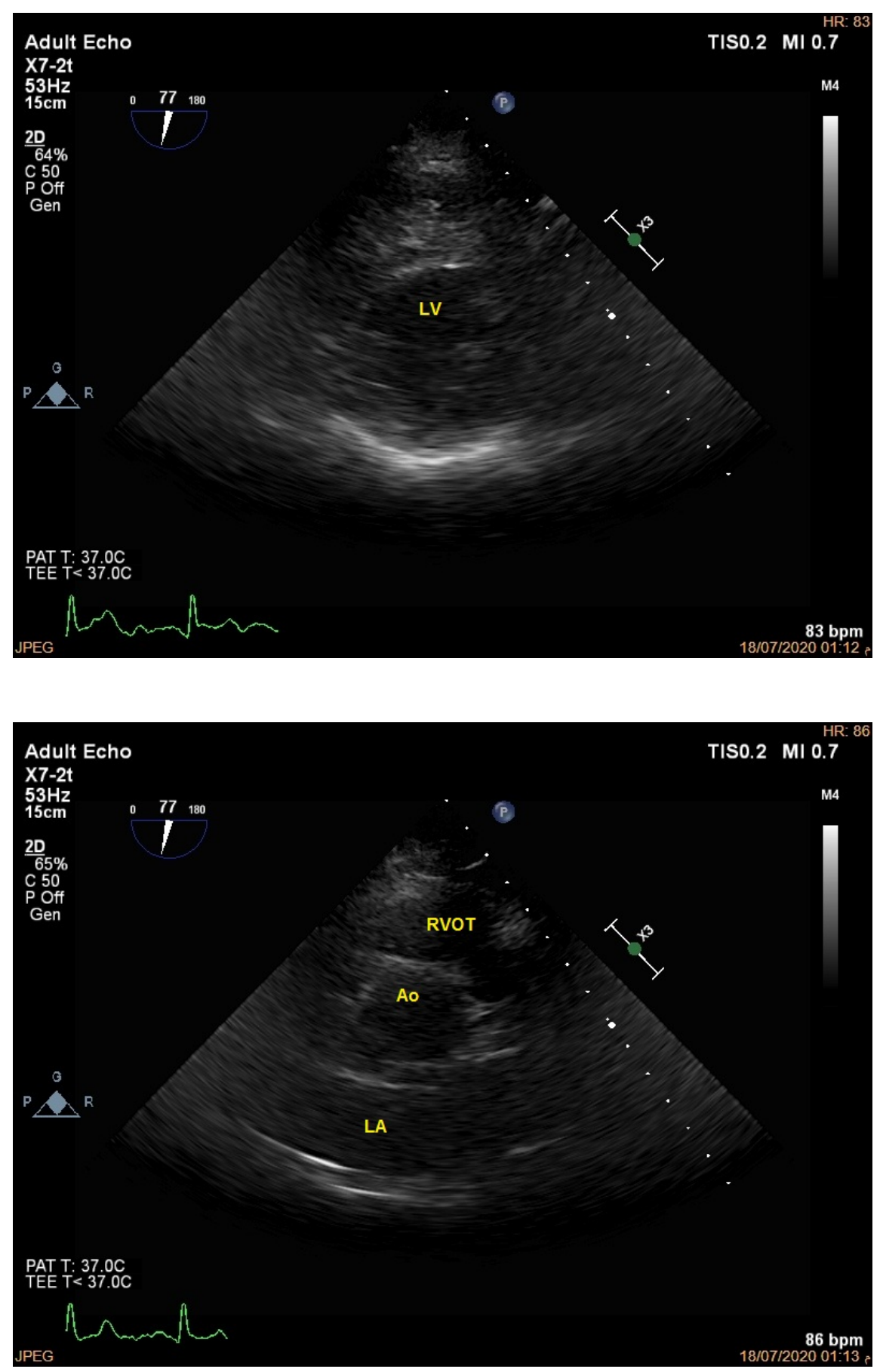
figures/figure-3/figure-3-eps-converted-to.pdf 
figures/Figure-4/Figure-4-eps-converted-to.pdf 
figures/Figure-5/Figure-5-eps-converted-to.pdf 
figures/Figure-6/Figure-6-eps-converted-to.pdf 
figures/Figure-7/Figure-7-eps-converted-to.pdf 
figures/Figure-8/Figure-8-eps-converted-to.pdf 